\title{
Micro-Focus X-Ray Fluorescence ( $\mu$-XRF) as an Extension of the Analytical SEM
}

\author{
V.-D. Hodoroaba,* V. Rackwitz, ${ }^{*}$ and D. Reuter* \\ * BAM Federal Institute for Materials Research and Testing, 12200 Berlin, Germany
}

The technological progress in the fields of small-spot low-power X-ray tubes and of polycapillary Xray optics has enabled the development of compact micro-focus X-ray sources that can be attached onto a SEM/EDS (scanning electron microscope with energy dispersive X-ray spectrometer) system [1-3], see Fig. 1. Thus, micro-focus X-ray fluorescence ( $\mu$-XRF) can be performed with an SEM, so that the analytical figures of merit available at the SEM are considerably extended. The implementation of $\mu-\mathrm{XRF}$ at SEM/EDS is especially attractive due to the possibility of using many options offered by the existing SEM/EDS systems, e.g. acquisition and identification of the X-ray fluorescence spectra. The sample stage control (existent at almost all modern SEMs) can also be used for carrying out X-ray fluorescence spectral maps in the manner that is well known in $\mathrm{SEM} / \mathrm{EDX}$.

By combining the analytical information obtained from the X-ray spectra excited with electrons and with photons respectively, the main elements as well as trace elements of low and high atomic number can be analyzed - with different spatial resolutions [4]. Relevant examples will be shown, see e.g. Fig. 2.

Commercial software packages for a quick reference-free quantification of X-ray fluorescence spectra are available [5]: WinAxil (Canberra), iMOXS-Quant (IFG Institute for Scientific Instruments), Vision (EDAX), iXRF (iXRF Systems), etc. This paper emphasizes not only the analytical benefits of the $\mu$-XRF with SEM/EDS but also the sources of significant inaccuracies residing in instrumental parameters such as: (i) excitation spectrum (X-ray tube spectra and real transmission of the X-ray optics), (ii) measurement geometry and (iii) X-ray spectrometer efficiency (up to $50 \mathrm{keV}$ ). Furthermore, procedures for the accurate determination of these parameters developed in the recent years in our lab will be presented.

Special attention is paid to the determination of the net peak intensities in the X-ray fluorescence spectra, i.e. to the reconstruction of the spectral background. For low atomic number matrices this is mainly caused by X-ray scattering in the specimen and is quite challenging to be described accurately with closed analytical formulae. Nevertheless, a model for scattering based on first principles was employed and verified by means of calibrated instrumentation and reference materials. The potential of the implementation of this physical background model for XRF quantification will be highlighted.

References

[1] B. J. Cross, K. C. Witherspoon, Microsc. Today 12 (2004) 20.

[2] A. Bjeoumikhov, V. Arkadiev, F. Eggert, V.-D. Hodoroaba, N. Langhoff, M. Procop, J. Rabe, R. Wedell, X-Ray Spectrom. 34 (2005) 493.

[3] M. Procop, V.-D. Hodoroaba, Microchim. Acta 161 (2008) 413.

[4] M. Haschke, F. Eggert, W. T. Elam, X-Ray Spectrom. 36 (2007) 254.

[5] J. Sherman, Spectrochim. Acta 7 (1955) 283. 
[6] This work is partly supported by the "BAM-Innovationsoffensive" program and "MNPQTransfer" project (BMWi, 23/07), respectively.

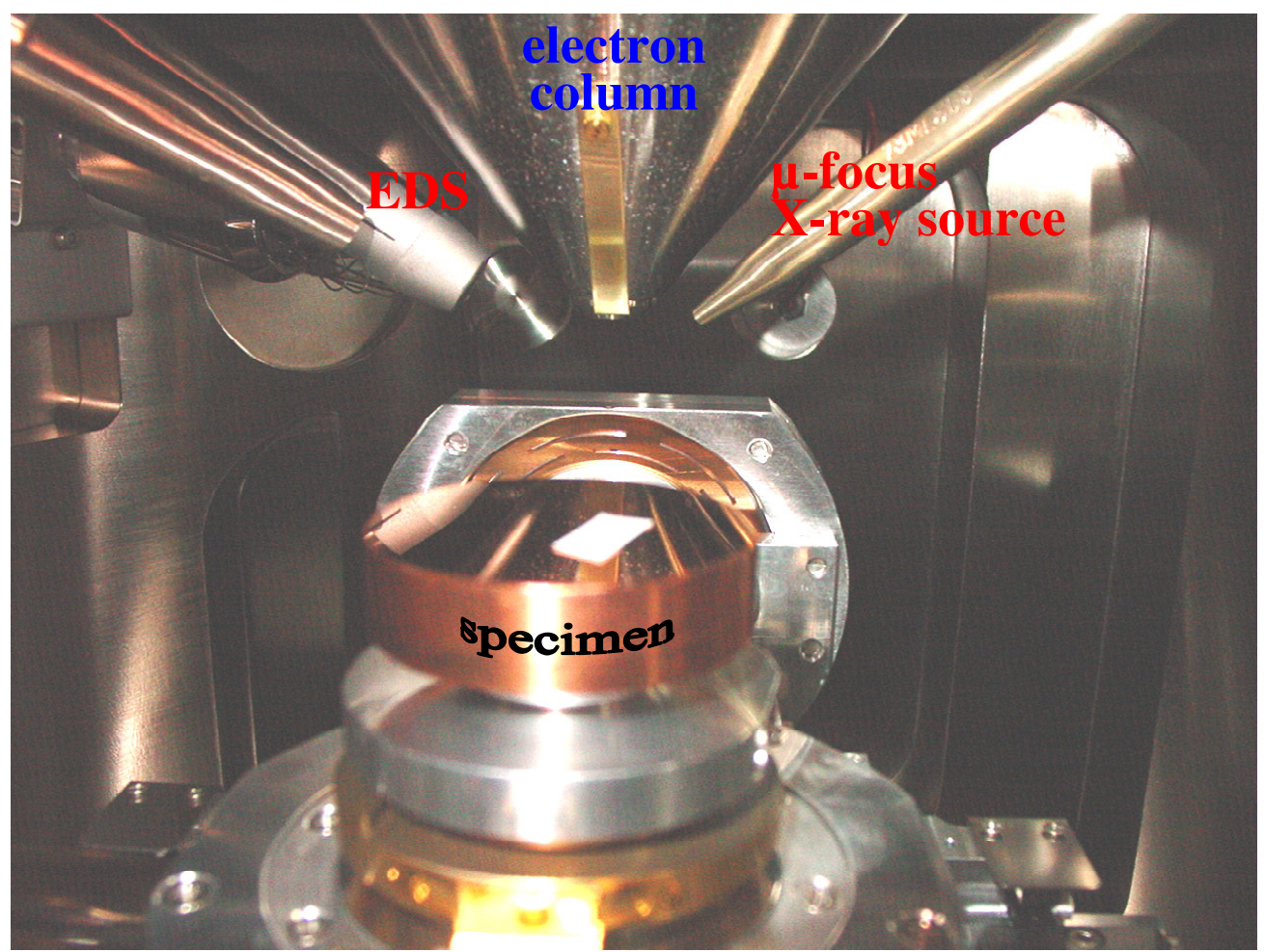

FIG. 1. Photograph of the SEM chamber showing the electron column, the $\mu$-focus X-ray source, the specimen and the EDS.
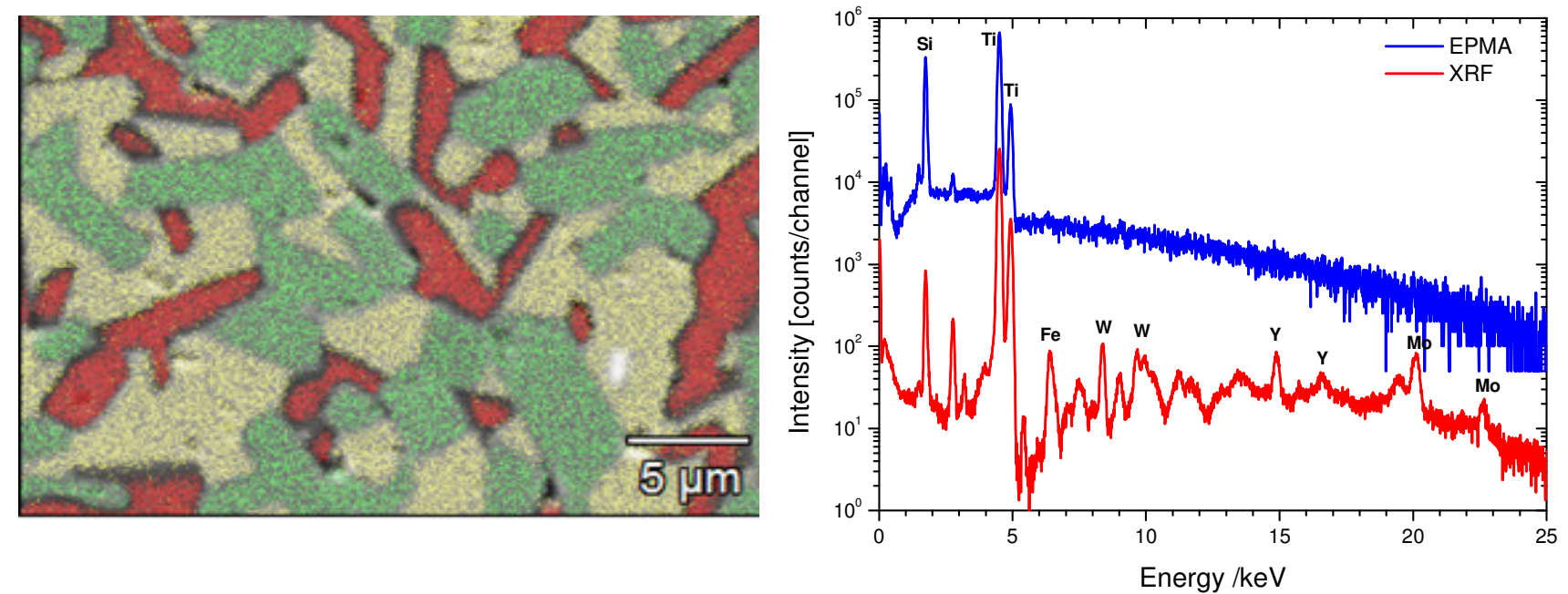

FIG. 2. Left: Electron excited elemental X-ray map of a composite ceramic indicating clearly three phases: $\mathrm{SiC}$ (red), $\mathrm{TiC}$ (yellow) and $\mathrm{TiB}_{2}$ (green). Right: electron excited X-ray spectrum (blue) of the sample range left and photon excited X-ray spectrum (red) revealing the presence of $\mathrm{Fe}, \mathrm{W}$ and $\mathrm{Y}$ at trace level concentration. 